\title{
MEES-WuR: Minimum Energy Coding with Early Shutdown for Wake-up Receivers
}

\author{
Nour El Hoda Djidi, Matthieu Gautier, Antoine Courtay and Olivier Berder
}

\begin{abstract}
One of the main challenges of wireless sensor networks is to maintain sensor nodes alive as long as possible, and a lot of efforts are dedicated to enable energy efficient communications. Wake-Up Receivers (WuRs) represent a promising solution for reducing the power consumption of nodes by enabling asynchronous communications. However, to achieve an ultra-low power consumption, WuRs circuits are kept as simple as possible, inducing a low sensitivity and thus a short range communication. As channel coding improves sensitivity, we propose to take advantage of the computing capability of the WuRs to apply a specific channel coding. The novelty resides in applying Minimum Energy coding with an Early Shutdown (MEES) of WuRs based on On-Off Keying (OOK) detectors. Both theoretical derivations and Monte-Carlo simulations show that the proposed coding scheme improves the reliability. Moreover, Moreover, MEES has been implemented on a non-coherent WuR prototype, and it is shown through experimentation that $\mathrm{WuR}$ reliability can be raised up to $22 \%$ compared to uncoded communications. Moreover, both the energy consumption and the latency can be significantly decreased thanks to the shutdown mechanism of MEES.
\end{abstract}

Index Terms-Energy efficiency, Minimum energy coding, Reliability, Wake-up receivers, Wireless sensor networks

\section{INTRODUCTION}

The deployment of several billions of nodes, thus forming the Internet of Things (IoT), allows to optimize many application fields such as smart cities, smart factories, e-health and smart grids [1]. IoT nodes provide sensing and actuating capabilities and are generally powered by batteries restricted in size and capacity [2], and have thus a limited lifetime. With the massive deployment of IoT devices, replacing batteries frequently will not only be costly but infeasible. Thus, extending the nodes lifetime is fundamental to keep them autonomous as long as possible.

In most applications, communication is the most power hungry task compared to other tasks (sensing, actuation and processing) [3]. Among the methods proposed to reduce energy consumption [4], [5], duty-cycling medium access protocols are traditionally adopted [4], where nodes are put in sleep mode by default and periodically woken up to receive or transmit data. However, this technique suffers from idle listening and overhearing, when a node senses the channel waiting for data and when a node overhears a packet that was not intended to it, respectively [6]. An alternative and promising solution for energy efficient wireless communication is the use of an Ultra Low Power (ULP) Wake-up Receivers (WuRs) [7].

N. Djidi, M. Gautier, A. Courtay and O. Berder are with Univ. Rennes, CNRS, IRISA, France
A WuR is an extra ULP receiver attached to the MicroController (MCU) of the main radio. It consumes typically a few hundreds of nano-watts of power whereas the main node consumes in the order of milli-watts, and it has lower bitrate than the main transceiver [8]. The WuR is continuously listening to the channel, while the main node is in sleep mode, allowing an asynchronous wake-up of the latter with a low latency [9]. When a specific signal called Wake-Up Beacon (WUB) is received, the WuR generates an interruption to wake-up the main node that performs afterwards data communication. Recent WuRs provide processing capabilities by embedding an ULP-MCU, which allows to perform an address decoding and activate only the specific main node according to the address received in the WUB [10].

The ULP consumption of the WuR is guaranteed by stringent constraints on the circuit architecture. Different circuit designs were reported in the literature and most of them are based on On-Off Keying (OOK) detectors [11]. ULP WuRs have a low sensitivity and thus can misdetect the received address in the WUB, leading to a range degradation of the communication system. Improving the sensitivity (e.g. through more sophisticated circuit design) would sacrifice the power consumption, thus a tradeoff between power consumption and sensitivity should be made. To deal with this problem, channel coding can be used as proposed in [12] and [13]. In the present paper, we propose a channel coding scheme that was never addressed for the WuR and that can fit the design of the WuR based on OOK. This channel coding is derived from Minimum Energy coding (ME) scheme [14], which is improved with an early shutdown capability of the ULP-MCU of the WuR. This new scheme, called Minimum Energy coding Early Shutdown (MEES), allows the WuR to improve its sensitivity, thus achieving a higher communication range while keeping energy efficiency. The main contributions of this work are:

- The application of ME coding scheme to WuRs for the first time with a novel technique to decode the WUB by applying MEES.

- The theoretical error probability derivation and energy consumption model of MEES for both coherent and noncoherent WuRs.

- The implementation of the MEES on a non-coherent $\mathrm{WuR}$, and the experimental evaluation of three essential metrics, the WUB Delivery Rate $(W D R)$, the average power consumption and the average latency.

The rest of this paper is organized as follows. In Section II, related works are presented. Section III details the principle of MEES coding and the bit error probability is derived. 
The energy consumption and latency models are given in Section IV. In Section V, the experimental evaluation of missed detection rate, $W D R$, average power consumption and average latency are presented before to conclude in Section VI.

\section{RELATED WORKS}

This section summarizes the related works relevant to the circuitry design of WuR and channel coding schemes that could be suitable to WuRs based on OOK detectors. For more detailed surveys on the state of the art of WuR designs, please refer to [11] and [15].

\section{A. Wake-up receivers}

The most popular modulation used for WuRs is OOK [11]. This simple modulation allows a simplification in the WuR circuitry and has therefore a low power consumption. The circuitry design can be based on non-coherent receivers (e.g. envelope detectors) or coherent receivers (e.g. mixer-based detectors). Non-coherent detectors are relatively simple compared with coherent receivers and are achieving low power consumption at the cost of a low sensitivity, while coherent detectors are improving the sensitivity but increasing the power consumption.

The idea of developing the WuR was first conceived by the PicoRadio project [16], which proposed a CMOS architecture that can be used either alone as a main radio on the sensor node, or as a wake-up radio. Using a frequency of $1.9 \mathrm{GHz}$ with bitrate up to $100 \mathrm{kbps}$, the proposed module can reach an indoor communication range of $20 \mathrm{~m}$, consuming $1.6 \mathrm{~mW}$ in transmit mode and $380 \mu \mathrm{W}$ in receive mode, with a receiver sensitivity of $-75 \mathrm{dBm}$.

Bryant et al. proposed in [17] a Coherent OOK (COOK) WuR based on uncertain-IF architecture, consuming $50 \mu \mathrm{W}$ at a frequency of $2.45 \mathrm{GHz}$ and achieving a sensitivity of $88 \mathrm{dBm}$ at a bitrate of $250 \mathrm{kbps}$. Thanh et al. proposed in [18] another COOK WuR design using uncertain-IF architecture that achieves a sensitivity of $-55 \mathrm{dBm}$ at a bitrate of $50 \mathrm{kbps}$, operating at a frequency of $430 \mathrm{MHz}$ while consuming $100 \mu \mathrm{W}$. Moazzeni et al. presented in [19] a COOK WuR with two different modes, a monitoring mode and an identifier mode for an operating frequency range of 902-928 $\mathrm{MHz}$. This WuR achieves a sensitivity of $-78.5 \mathrm{dBm}$ and $-75 \mathrm{dBm}$ while consuming an average power of $16.4 \mu \mathrm{W}$ and $22.9 \mu \mathrm{W}$ using the two modes, respectively.

Bdiri et al. proposed in [20] a WuR based on a NonCoherent OOK (NCOOK) detector that consumes less than $3 \mu \mathrm{A}$ with a sensitivity of $-60 \mathrm{dBm}$. Magno et al. proposed in [21] a WuR with a passive NCOOK envelope detector. This WuR works at a bitrate of $1 \mathrm{kbps}$ with a sensitivity of $-55 \mathrm{dBm}$ and a frequency of $868 \mathrm{MHz}$. The power consumption of the whole WuR was measured to be $1.83 \mu \mathrm{W}$ in always on listening mode, while the ULP-MCU is turned off, and $284 \mu \mathrm{W}$ when processing the data with the ULP-MCU.

More recent circuits were developed achieving a higher sensitivity. Wang et al. proposed in [22] a WuR based on NCOOK detector consuming $6.1 \mathrm{nW}$ with a sensitivity of $-80.5 \mathrm{dBm}$. Moody et al. proposed in [23] a NCOOK WuR achieving a sensitivity of $-76 \mathrm{dBm}$ at a frequency of $151.8 \mathrm{MHz}$, and a bitrate of $200 \mathrm{~b} / \mathrm{s}$ with a total power consumption of $7.6 \mathrm{nW}$. Furthermore, Jiang et al. proposed in [24] a temperature-robust NCOOK-based WuR consuming $22.3 \mathrm{nW}$ at $9 \mathrm{GHz}$ with a sensitivity of $-69.5 \mathrm{dBm}$ and a bitrate of $33.3 \mathrm{~b} / \mathrm{s}$.

The tradeoff between sensitivity and power consumption clearly depends on the detector architecture, the operating frequency and the bitrate. Despite design improvements, WuR sensitivity still lags that of most main radios, and can be further improved using its processing capability for any given design. This is why we will investigate the use of channel coding that improves the sensitivity of OOK-based WuR while being energy efficient.

\section{B. Channel coding}

Channel coding involves adding extra (redundant) bits to the data stream so that the decoder can detect or even correct errors at the output of the receiver and thus improve its sensitivity [25]. Different channel coding exist in the literature such as Hamming Walsh, turbo and Low-Density Parity Check (LDPC) codes. Complex codes may provide better performance but at the cost of high power consumption and long latency due to the decoding complexity such as LDPC [26]. Therefore, when considering applications for WSNs, simple but efficient codes are more interesting. Channel coding schemes suitable for OOK detectors in general are first investigated, then those proposed for WuRs are evoked in this Section.

1) Channel coding for OOK detectors: Zhang et al. [27] investigated the capacity behavior of NCOOK schemes over an Additive White Gaussian Noise (AWGN) channel at low Signal to Noise Ratio (SNR). The study demonstrates that NCOOK based on soft and hard decisions can achieve the Shannon limit with asymmetric 'on off' distributions. In addition, they suggest that Pulse-Position Modulation (PPM) based schemes could be suitable channel coding schemes combined with OOK signaling due to the asymmetric distribution of ones and zeros within a PPM symbol. In PPM coding, the information is in the position of the one.

Tanget et al. studied in [14] the Bit Error Rate (BER) performance of the OOK based ME coding, that is a sort of PPM coding, for both coherent and non-coherent circuits and with both hard and soft decisions. The study obviously shows that ME coding outperforms uncoded scheme, but also that non-coherent ME coding is slightly degraded from coherent ME coding.

2) Channel coding applied to WuRs: Combining WuR and channel coding has not been exhaustively addressed in the literature. Mazloum et al. proposed in [28] to spread each bit of the address in the WUB by an arbitrary code. The study shows an improvement of the BER but at the cost of a high code length. For example, they propose to spread each bit into a code of 15 bits, thus an address of 4 bits requires 60 bits once being encoded, which will drastically increase the latency especially when low bit rate WuRs are used. Ghose et al. proposed in [12] to transmit a small amount of data by adding a Cyclic Redundancy Check (CRC). This CRC concerns only 
the data and not the address sent in the WUB, and thus if an error occurs during the transmission of the address contained in the WUB, it can not be detected.

Rakovic et al. proposed in [13] to add error correction codes to the WUB, comparing the performance of three coding schemes: Walsh, repetition coding and Hamming code. Walsh code achieves the best performance, but it requires a significantly large addressing size (32B) and high memory, that is practically impossible. Moreover, Hamming achieves the worst performance of all coding approaches, with a probability of error comparable to the uncoded case. Furthermore, no experimental evaluation was done.

\section{Minimum ENERGY CODING EARLY SHUTDOWN}

Before presenting MEES principle, the WuR system considered in this work is detailed, then a bit error probability model is proposed and evaluated.

\section{A. WuR system design}

The WuR is composed of two parts, an analog front-end that contains a signal rectifier and a digital part composed of an ULP-MCU as it can be shown in Fig. 1(a). The analog front-end is always active while consuming a few nano-watts, contrary to the ULP-MCU that is kept off. When a source node has data to send to a destination node, it first sends a WUB. The WUB contains a preamble and the address of the destination as illustrated in Fig. 1(b). The preamble consists of few bits at ' 1 ' allowing to trigger the ULP-MCU from the sleep state, thus increasing the power consumption. Once the ULP-MCU is triggered on, it starts decoding the received address to wake-up the main node only if it is the destination node.

\section{B. MEES principle and its application to the WuR}

1) Principle: The basic idea of $\mathrm{ME}$ coding consists in mapping every $k$ bits of a source bitstream into a $n$-bits codeword as shown in TABLE I. The all-zeros source symbol $S_{0}$ is mapped into $n$-bits all-zeros codeword $M_{0}$. All other source symbols $S_{i}$ are mapped into $n$ bits codewords $M_{i}$ with only one ' 1 ' bit, the $i^{t h}$ bit in the codeword. The codeword of size $n$ contains in total $2^{k}-1$ bits.

TABLE I

MinimuM ENERGY CODING MAPPING TABLE.

\begin{tabular}{|c|c|c|c|}
\hline \multicolumn{2}{|c||}{ Source symbols $(k)$} & \multicolumn{2}{c|}{ Mapped symbols $\left(n=2^{k}-1\right)$} \\
\hline$S_{0}$ & $00 . .00$ & $M_{0}$ & $0000 . .000$ \\
$S_{1}$ & $00 . .01$ & $M_{1}$ & $0000 . .001$ \\
$\ldots$ & $\ldots$ & $\ldots$ & $\ldots$ \\
$\ldots$ & $\ldots$ & $\ldots$ & $\ldots$ \\
$\ldots$ & $\ldots$ & $\ldots$ & $\ldots$ \\
$S_{n-1}$ & $11 . .10$ & $M_{n-1}$ & $0100 . .000$ \\
$S_{n}$ & $11 . .11$ & $M_{n}$ & $1000 . .000$ \\
\hline
\end{tabular}

ME coding is a kind of PPM coding. The only difference is when the symbol is all zero, it is mapped to all-zero codeword in ME coding, while in PPM coding it is mapped to one pulse in the first position. Thus, in PPM coding a symbol of $k$ bits is mapped in a codeword consisting of a single binary one and $2^{k}-1$ binary zeros resulting in a codeword of $2^{k}$ bits. Besides, with $\mathrm{ME}$ coding it is mapped to $2^{k}-1$ bits.

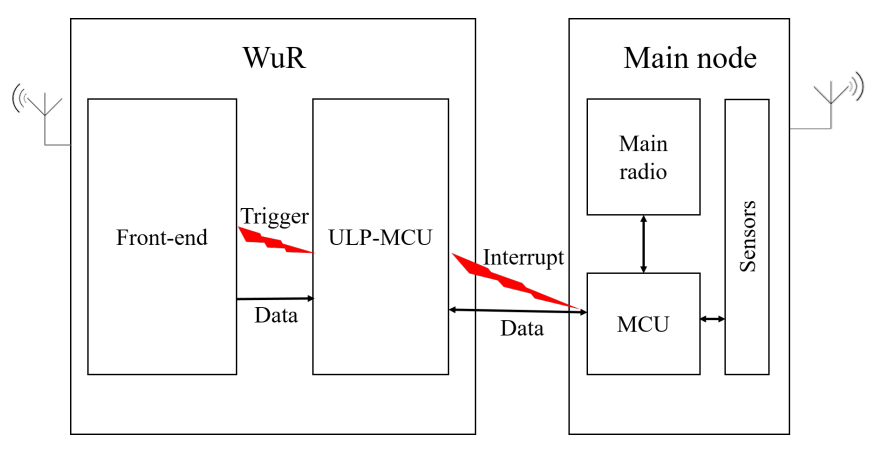

(a) WuR block diagram

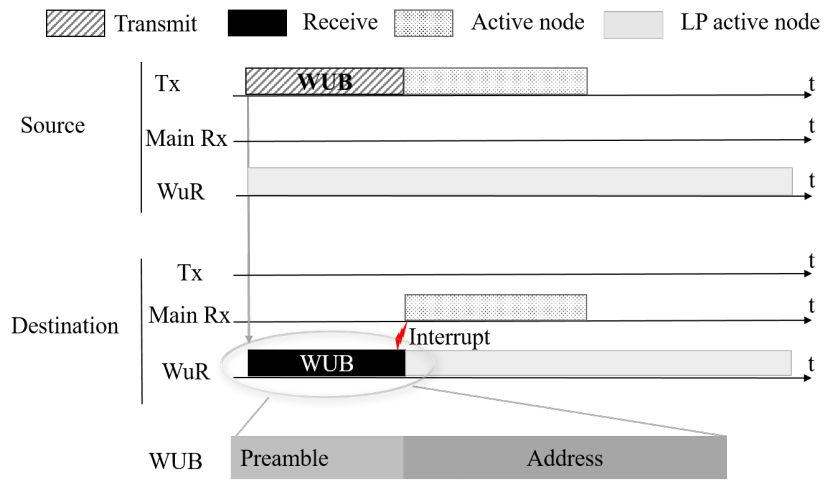

(b) Mechanism of the WUB exchange between source and destination nodes

Fig. 1. WuR system design.

2) Application of MEES to the WuR: The WuR is always listening to the channel while the ULP-MCU is in deep sleep and turned on once the WUB is received in order to decode the address. We consider a transmitter that turns off its power amplifier when sending the '0', and as the codeword contains more zeros than ones, a significant amount of energy can be saved. However, on the WuR side, the latency and energy consumption will increase as the length of the codeword is longer compared to the uncoded symbol. In order to save energy and reduce this latency, we propose a novel scheme when receiving the WUB. As the codeword contains one bit at ' 1 ' and all the others at ' 0 ', we propose to shutdown the ULP-MCU as soon as the first ' 1 ' is received (the address information is directly given through the position of the '1'). This novel technique is called Minimum Energy coding Early Shutdown (MEES). The difference between ME and MEES is illustrated on Fig. 2. It can be seen that in case of $\mathrm{ME}$ coding (Fig. 2(a)), the ULP-MCU stays active during the whole duration of the WUB. However, with MEES (Fig. 2(b)) the ULP-MCU is turned off as soon as the first '1' is received in the WUB, thus with MEES the latency and the energy consumed by the reception of the WUB depends on the position of the bit at ' 1 '.

\section{MEES bit error probability}

An investigation of the impact of the MEES on the bit error probability compared to ME for both NCOOK and COOK is performed in this Section. For that, it is necessary to reproduce the bit error probability of ME coding to derive that of MEES. 

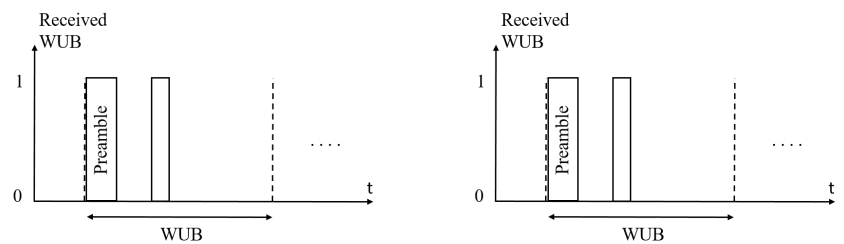

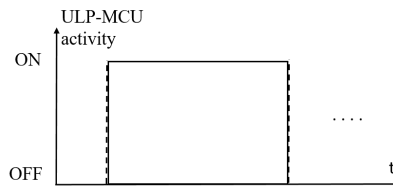

(a) ME scheme

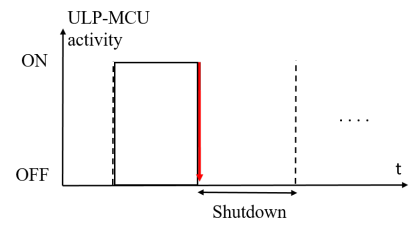

(b) MEES scheme
Fig. 2. WUB reception example with ME and MEES.

Assuming that a source symbol $S_{i}$ is transmitted using the codeword $M_{i}$, the receiver takes a hard decision on each bit. The result can be:

- No error, the codeword $M_{i}$ is received and decoded to the correct source symbol $S_{i}$

- A wrong codeword $M_{j}$ is received resulting in a source symbol $S_{j}$

- An illegal codeword $M_{b}$ is received (i.e. multiple '1' instead of only one bit at '1'), meaning that a random source symbol is taken in case of ME coding [14]. On the other hand, with MEES the first '1' received is taken, as the ULP-MCU will be shutdown right after the reception of the first ' 1 '.

The theoretical bit error probability $P_{e}$ of $\mathrm{NCOOK}$ and COOK receiver over an AWGN channel is:

$$
P_{e}=\frac{1}{2}\left(P_{e S}+P_{e M}\right)
$$

where $\mathrm{P}_{e S}$ and $\mathrm{P}_{e M}$ are the error probabilities of '0' being received as '1' and '1' being received as '0', respectively.

For a NCOOK receiver, $\mathrm{P}_{e S}$ and $\mathrm{P}_{e M}$ are expressed as [29]:

$$
P_{e S}=\exp \left(\frac{-b^{2}}{2 \sigma^{2}}\right)
$$

and

$$
P_{e M}=1-Q\left(\sqrt{\frac{2 E_{b}}{N_{0}}}, \frac{b}{\sigma}\right),
$$

with $\mathrm{E}_{b} / \mathrm{N}_{0}$ the energy-per-bit to noise-density ratio, $\sigma$ the variance of additive white noise at the input of the envelope detector, $b$ the decision threshold defined as:

$$
b=\sigma \sqrt{2+\frac{E_{b}}{2 N_{0}}},
$$

and $Q($.$) the Marcum- Q$ function defined as:

$$
Q(u, v)=\int_{v}^{\infty} \exp \left(-\frac{u^{2}+x^{2}}{2}\right) I_{0}(u x) x d x,
$$

with $I_{0}$ the modified Bessel function of the first kind of zero order.
For a COOK receiver $\mathrm{P}_{e S}$ and $\mathrm{P}_{e M}$ are expressed as [29]:

$$
P_{e S}=P_{e M}=Q\left(\sqrt{\left(E_{b} / 2 N_{0}\right)}\right) .
$$

The theoretical bit error probability when applying $\operatorname{ME}(n, k)$ has been expressed in [14] and will be adjusted for the $\operatorname{MEES}(n, k)$ scheme.

When applying $\operatorname{MEES}(n, k)$ or $\operatorname{ME}(n, k)$, there are two different situations, original symbol is not all-zero and original symbol is all-zero.

1) Original symbol $S_{i}$ is not all-zero

- The probability to get the correct symbol $S_{i}$ is:

$$
P_{i i}=\left(1-P_{e M}\right)\left(1-P_{e S}\right)^{(n-1)},
$$

with the bit error probability equals to zero as there is no error.

- The probability to get another symbol $S_{j}$ is:

$$
P_{i j}=P_{e M} P_{e S}\left(1-P_{e S}\right)^{(n-2)},
$$

with, in this case the bit error probability is $\frac{d_{i j}}{k}$, with $d_{i j}$ the number of bit errors representing the Hamming distance $d_{i j}$ between symbols $S_{i}$ and $S_{j}$.

- The probability to get all-zero symbol $S_{0}$ is:

$$
P_{i 0}=P_{e M}\left(1-P_{e S}\right)^{(n-1)},
$$

where the bit error probability is $\frac{d_{i 0}}{k}$.

- The probability to get an illegal word is:

$$
P_{i b}=1-P_{i 0}-P_{i i}-\sum_{j \neq 0, i}^{n} P_{i j},
$$

and the average bit error probability $P_{i b e}$ is:

$$
P_{i b e}=\frac{1}{n+1} \sum_{i=0}^{n} \frac{d_{i j}}{k}=\frac{1}{2},
$$

and

$$
\sum_{j=0}^{n} d_{i j}=k 2^{k-1}, \forall i \in[0, n] .
$$

When receiving an illegal codeword with MEES, as the ULP-MCU is turned off as soon as the first '1' is received, there is no probability to decode an illegal codeword as a symbol '000.....00' or '000.....001'. Considering all possible symbols that could be sent and all possible symbols that could be received in case of an illegal codeword, we get:

$$
\begin{gathered}
\sum_{i=0}^{n} \sum_{j \neq 0, j \neq 1}^{n} d_{i j}=k 2^{k}\left(2^{k-1}-1\right) . \\
\sum_{i=0}^{n} d_{i j}=\frac{1}{2^{k}-2} \sum_{i=0}^{n} \sum_{j \neq 0, j \neq 1}^{n} d_{i j}=k 2^{k-1} .
\end{gathered}
$$

Thus the average bit error probability when using MEES coding is the same as using ME coding. 
The average bit error probability when not all-zero symbol is transmitted with either ME or MEES is:

$$
P_{e / S_{i}}=P_{i 0} \frac{d_{i j}}{k}+\sum_{j \neq 0, i}^{n} P_{i j} \frac{d_{i j}}{k}+\frac{P_{i b}}{2} .
$$

2) Original symbol $S_{i}$ is all-zero

- The probability to get the correct symbol $S_{0}$ is:

$$
P_{00}=\left(1-P_{e S}\right)^{n},
$$

with the bit error probability equals to zero.

- The probability to get another symbol $S_{j}$ is:

$$
P_{0 j}=P_{e S}\left(1-P_{e S}\right)^{n-1},
$$

in this case the bit error probability is $\frac{d_{0 j}}{k}$.

- The probability to get an illegal word is:

$$
P_{0 b}=1-P_{00}-\sum_{j \neq 0}^{n} P_{0 j},
$$

and as described in (11) the bit error probability is $\frac{1}{2}$. Then the average bit error probability when the all-zero symbol is transmitted is:

$$
P_{e / S_{0}}=\sum_{j \neq 0}^{n} P_{0 j} \frac{d_{0 j}}{k}+\frac{P_{0 b}}{2}=P_{e S}\left(1-P_{e S}\right)^{n-1} \sum_{j=1}^{n} \frac{d_{0 j}}{k}+\frac{P_{0 b}}{2} .
$$

Finally with (14) and (18), and the assumption of uniform distribution of source symbols, the average bit error probability when applying MEES or ME is expressed as:

$$
\begin{aligned}
P_{e} & =\frac{1}{n+1} \sum_{i=1}^{n} P_{e / S_{i}}+P_{e / S_{0}} \\
& =\frac{1}{n+1}\left[\frac{n+1}{2} P_{e M}+\frac{n+3}{2} P_{e S}-P_{e M} P_{e S}\right. \\
& \left.-P_{e S}^{2}-\frac{1}{2}(n+1)\right]\left(1-P_{e S}\right)^{n-2}+\frac{1}{2},
\end{aligned}
$$

where $P_{e S}$ and $P_{e M}$ are given by (2a) and (2b), respectively in case of NCOOK, whereas in case of COOK $P_{e S}=P_{e M}$ and it is given by (5).

\section{BER evaluation of non-coherent WuR}

If $E_{b}$ is the energy used to send one modulation bit and $E_{a v}$ is the average energy used to send a source data bit, for OOK, $E_{a v}=\frac{E_{b}}{2}$. When using ME coding, a $k$-bit non-zero source symbol is transmitted as $n$-bit codeword with only one bit at '1'. An all-zero source symbol does not consume energy. The average energy consumed is:

$$
E_{a v}=\frac{E_{b}}{k} \frac{n}{n+1} .
$$

Fig. 3(a) compares the bit error probability obtained from (1) and (19), and the BER obtained from monte-carlo simulations (circle symbols) as a function of the average bit energy to

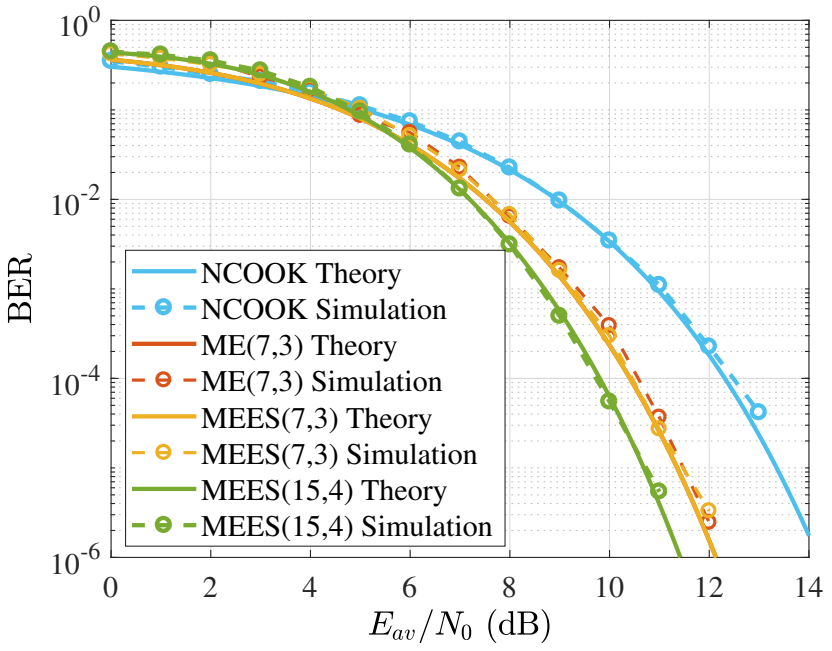

(a) BER for a non-coherent WuR

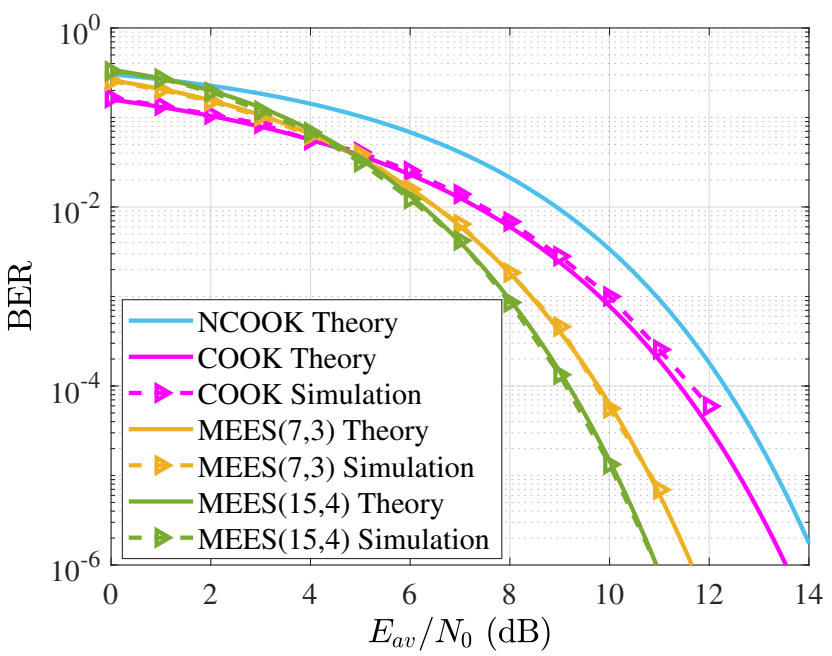

(b) BER for a coherent WuR

Fig. 3. BER as a function of $\mathrm{E}_{a v} / \mathrm{N}_{0}$.

noise ratio $\mathrm{E}_{a v} / \mathrm{N}_{0}$ at the receiver input for a NCOOK receiver, and when applying $\operatorname{ME}(7,3), \operatorname{MEES}(7,3)$ and $\operatorname{MEES}(15,4)$.

For figure clarity, the simulation and theoretical results of both $\operatorname{ME}(7,3)$ and $\operatorname{MEES}(7,3)$ are represented, while when $k=4$, only MEES is represented. It can be seen that the simulation matches with the theoretical derivation confirming the theoretical results derived for MEES, and thus the BER when using ME coding is the same as MEES coding.

A coding gain is defined as the reduction in $\mathrm{E}_{a v} / \mathrm{N}_{0}$ that is achieved when coding is used, when compared with the $\mathrm{E}_{a v} / \mathrm{N}_{0}$ required for the uncoded case at some specific level of BER. It can be seen that with a BER of $10^{-4}$ a coding gain of $2 \mathrm{~dB}$ and $2.5 \mathrm{~dB}$ is achieved with $\operatorname{MEES}(7,3)$ and $\operatorname{MEES}(15,4)$, respectively.

We can also see that with $\mathrm{E}_{a v} / \mathrm{N}_{0}$ of $12 \mathrm{~dB}$, the BER is equal to $2.6 \times 10^{-4}$ without coding and can be reduced to $3.6 \times 10^{-6}$ and $3.9 \times 10^{-7}$ when the $\operatorname{MEES}(7,3)$ and $\operatorname{MEES}(15,4)$ are used, respectively, thus with the same transmission power, we can get a lower BER when MEES coding is used. MEES coding can either achieve better BER under the same energy consumption or achieve energy efficiency under the same 
BER.

The figure also shows that there is a coding threshold in the sense that the coded system provides poorer performance than the uncoded system when $\mathrm{E}_{a v} / \mathrm{N}_{0}$ is less than the threshold value. A coding threshold is found in all coded systems [14] and it is equal to $5 \mathrm{~dB}$ when applying both $\operatorname{MEES}(7,3)$ and $\operatorname{MEES}(15,4)$.

\section{E. BER evaluation of coherent WuR}

Fig. 3(b) shows the BER as a function of the average bit energy to noise ratio $\mathrm{E}_{a v} / \mathrm{N}_{0}$ at the receiver input for a COOK receiver. It can be seen that when the BER is equal to $10^{-4}$ a coding gain of $0.5 \mathrm{~dB}$ is achieved compared to the NCOOK WuR. Furthermore, when applying $\operatorname{MEES}(7,3)$ and MEES(15,4), a gain of $1.6 \mathrm{~dB}$ and $2.2 \mathrm{~dB}$ is obtained, respectively, compared to uncoded coherent OOK. When comparing NCOOK in Fig. 3(a) and COOK in Fig. 3(b), it appears that when using respectively $\operatorname{MEES}(7,3)$ and $\operatorname{MEES}(15,4)$, a gain of $0.5 \mathrm{~dB}$ and $0.6 \mathrm{~dB}$ is achieved by the coherent receiver at $\mathrm{BER}=10^{-4}$.

The performance of coherent WuR is slightly improved, but non-coherent WuR has the benefits of its simplicity and lower power consumption which can compensate for the small performance deterioration.

\section{ENERGY CONSUMPTION AND LATENCY MODELS}

\section{A. Network scenario and assumptions}

A one-hop network is considered with $N$ sensor nodes in which all nodes are within the WuR range of each other. Each node generates $\lambda$ packets/s, and thus sends the same number of packets to one of its neighbors with equal chance [30]. No collision is considered in our energy consumption model as the network is not so dense due to limited range of wake-up radio and packet generation rate is rather low. The generated packets are assumed to be unicast with identical length.

\section{B. Energy consumption model}

The model of the average total power consumption of the node is derived by considering all the states that the node passes through. For the model clarity, the average power consumption of transmitting the WUB and the average power consumption of the WuR according to the used coding scheme are first derived, and then the average total power consumption of the node is given.

1) Average power consumption without coding: The average power consumption $\bar{P}_{t x}^{\text {uncoded }}$ to successfully transmit a WUB with an equal probability to transmit ' 1 ' and ' 0 ' is expressed as:

$$
\bar{P}_{t x}^{\text {uncoded }}=n_{t x} \lambda T_{b} P_{t x_{1}}\left(l_{\text {preamble }}+\frac{k}{2}\right)+n_{t x} \lambda T_{b} P_{t x_{0}}\left(\frac{k}{2}\right),
$$

where $P_{t x_{1}}$ and $P_{t x_{0}}$ are the power consumed to send ' 1 ' and ' 0 ', respectively, $T_{b}$ is the bit duration, $l_{\text {preamble }}$ is the number of bits used in the preamble, $k$ is the number of bits used in the address and $n_{t x}$ is the number of times the WUB is transmitted to be successfully received, and it is expressed as:

$$
n_{t x}=\frac{1}{W D R} .
$$

When a node transmits $\lambda$ packets/s to one of its neighbors, the $N-2$ other neighbors will overhear this WUB as they are in the WuR range of each others. Therefore, at each period of $\frac{1}{\lambda}$, the WuR will receive $N-1$ WUBs (the WUB that is intended to it and the $N-2$ from the overhearing). Therefore, the average power consumed by the WuR $\bar{P}_{W u R}^{\text {uncoded }}$ is:

$$
\begin{aligned}
\bar{P}_{W u R}^{\text {uncoded }} & =n_{t x}(N-1) \lambda T_{b}\left(l_{\text {preamble }}+k\right) P_{r x}^{\text {wur }} \\
& +\left(1-n_{t x}(N-1) \lambda T_{b}\left(l_{\text {preamble }}+k\right)\right) P_{\text {idle }}^{\text {wur }},
\end{aligned}
$$

with $P_{r x}^{w u r}$ the power consumed by the WuR when processing the received WUB, and $P_{i d l e}^{\text {wur }}$ the power consumed by the WuR when only the front end is active while the ULP-MCU is sleeping.

2) Average power consumption with $M E(n, k)$ : The average power consumption to successfully transmit the WUB when using $\operatorname{ME}(n, k)$ denoted $\bar{P}_{t x}^{M E}$ is expressed as:

$\bar{P}_{t x}^{M E}=n_{t x} \lambda T_{b} P_{t x_{1}}\left(l_{\text {preamble }}+\frac{n}{n+1}\right)+n_{t x} \lambda T_{b} P_{t x_{0}}\left(\frac{n^{2}}{n+1}\right)$.

The average power consumed by the WuR when using $\operatorname{ME}(n, k)$ denoted $\bar{P}_{W u R}^{M E}$ is:

$$
\begin{aligned}
\bar{P}_{W u R}^{M E} & =n_{t x}(N-1) \lambda T_{b}\left(l_{\text {preamble }}+n\right) P_{r x}^{w u r} \\
& +\left(1-n_{t x}(N-1) \lambda T_{b}\left(l_{\text {preamble }}+n\right)\right) P_{\text {idle }}^{\text {wur }} .
\end{aligned}
$$

3) Average power consumption with MEES(n,k): When ME coding is used with a shutdown of the ULP-MCU while

\begin{tabular}{|c|c|c|}
\hline MEES(n,k) & $l_{M E E S}$ & Energy consumed by a received codeword \\
\hline $00 \ldots \ldots \ldots 00$ & $n$ & $P_{r x}^{w u r} \times n \times ?$ \\
\hline $00 \ldots \ldots . .01$ & $n$ & $P_{r x}^{w u r} \times n \times T_{b}$ \\
\hline $00 \ldots \ldots \ldots 10$ & $n-1$ & $P_{r x}^{w u r} \times(n-1) \times T_{b}+P_{\text {idle }}^{\text {wur }} \times T_{b}$ \\
\hline . & . & \\
\hline . & . & \\
\hline . & . & \\
\hline $01 \ldots \ldots . . .00$ & 2 & $P_{r \times}^{w u r} \times 2 \times T_{b}+P_{i}^{w u r} \times(n-2) \times T_{b}$ \\
\hline $10 \ldots \ldots .00$ & 1 & $P_{r x}^{w u r} \times 1 \times T_{b}+P_{i d l e}^{w w r} \times(n-1) \times T_{b}$ \\
\hline
\end{tabular}
receiving the first ' 1 ', the energy consumed by the reception of the WUB, that depends on the position of the bit at ' 1 ' denoted $l_{M E E S}$, is given in TABLE II.

TABLE II

ENERGY CONSUMPTION PER CODEWORD IN EARLY SHUTDOWN MODE.

Let $\bar{l}_{M E E S}$ be the average position of the bit at ' 1 ', it is expressed as:

$$
\bar{l}_{M E E S}=\frac{1}{n+1}\left(2 n+\frac{n(n-1)}{2}\right)=\frac{n(n+3)}{2(n+1)} .
$$

The average power consumption of the WuR when receiving the WUB based MEES denoted $\bar{P}_{W u R}^{M E E S}$ is expressed as: 


$$
\begin{aligned}
\bar{P}_{W u R}^{M E E S} & =\left[\left(l_{\text {preamble }}+\frac{1}{n+1}\left(2 n+\frac{n(n-1)}{2}\right)\right) P_{r x}^{w u r}\right. \\
& \left.+\frac{1}{n+1} \frac{n(n-1)}{2} P_{\text {idle }}^{w u r}\right] n_{t x}(N-1) \lambda T_{b} \\
& +\left(1-n_{t x}(N-1) \lambda T_{b}\left(n+l_{\text {preamble }}\right)\right) P_{i d l e}^{w u r} \\
& =n_{t x}(N-1) \lambda T_{b}\left(l_{\text {preamble }}+\bar{l}_{M E E S}\right) P_{r x}^{w u r} \\
& +\left(1-n_{t x}(N-1)\left(l_{\text {preamble }}+\bar{l}_{M E E S}\right) \lambda T_{b}\right) P_{\text {idle }}^{\text {wur }}
\end{aligned}
$$

4) Average total power consumption of the node: The average total power consumption of the node that exchanges the WUB and the data depends on the used coding scheme. The node sends the WUB until it is successfully received by one of its neighbors, and then transmits the data and receives an acknowledgement from its neighbors. The average total power consumption of the node can be expressed as:

$$
\bar{P}_{\text {total }}=\bar{P}_{t x}^{x}+\bar{P}_{t x}^{\text {data }}+\bar{P}_{W u R}^{x}+\bar{P}_{r x}^{\text {data }}+\bar{P}_{\text {sleep }},
$$

with $\bar{P}_{t x}^{x}$ can be, according to the used coding scheme, either $\bar{P}_{t x}^{\text {uncoded }}$ or $\bar{P}_{t x}^{M E}$ defined in (21) or (24), $\bar{P}_{W u R}^{x}$ can be either $\bar{P}_{W u R}^{u n c o d e d}, \bar{P}_{W u R}^{M E}$ or $\bar{P}_{W u R}^{M E E S}$, defined in (23), (25) or (27), $\bar{P}_{t x}^{\text {data }} / \bar{P}_{r x}^{\text {data }}$ are the average power consumptions of transmitting/receiving a data to/from one of the neighbor with the main transceiver, $\bar{P}_{\text {sleep }}$ is the average power consumption of the sleep state of the main transceiver. $\bar{P}_{t x}^{d a t a}$ and $\bar{P}_{r x}^{\text {data }}$ are expressed as:

$$
\begin{aligned}
& \bar{P}_{t x}^{\text {data }}=\lambda\left(P_{t x} T_{\text {data }}+P_{r x} T_{a c k}\right), \\
& \bar{P}_{r x}^{\text {data }}=\lambda\left(P_{r x} T_{\text {data }}+P_{t x} T_{a c k}\right),
\end{aligned}
$$

with $P_{t x}$ the power consumption of transmitting with the main transceiver, $P_{r x}$ the power consumption of receiving with the main transceiver, $T_{\text {data }}$ the duration of data, and $T_{a c k}$ the duration of the acknowledgement. $\bar{P}_{\text {sleep }}$ is expressed as:

$$
\bar{P}_{\text {sleep }}=P_{s}\left(1-\lambda\left(2 T_{\text {data }}+2 T_{\text {ack }}+n_{t x} T_{w u b}\right)\right),
$$

with $P_{s}$ the power consumption in sleep state, $T_{w u b}$ the duration of transmitting the WUB that is equal $T_{w u b}=$ $T_{b}\left(l_{\text {preamble }}+k\right)$ in case of uncoded scheme, and it is equal to $T_{w u b}=T_{b}\left(l_{\text {preamble }}+n\right)$ in case of ME or MEES schemes.

\section{Latency model}

The average latency can be defined as the delay from receiving the WUB until sending the interrupt in case of receiving the correct address. The average latency in this case includes the duration of the preamble and the duration of decoding the address. It is also interesting to consider the total latency of successfully receiving the WUB followed by the data exchange in order to show the total latency gain brought by the proposed early shutdown mechanism. Therefore, the average latency of the WUB exchange is first derived according to the used coding scheme, and then the total WUB and data exchange latency is given.

\section{1) Average latency of the WUB exchange:}

- Average latency without coding: During the reception of the WUB, the ULP-MCU should wait to receive the whole address and check its correctness before sending the interrupt to the main node. The average latency without coding denoted $\bar{L}_{\text {uncoded }}$ is expressed as:

$$
\bar{L}_{\text {uncoded }}=T_{b}\left(l_{\text {preamble }}+k\right) .
$$

- Average latency with $\operatorname{ME}(n, k)$ : The average latency when using the ME coding denoted $\bar{L}_{M E}$ is expressed as:

$$
\bar{L}_{M E}=T_{b}\left(l_{\text {preamble }}+n\right) .
$$

- Average latency with $\operatorname{MEES}(\mathrm{n}, \mathrm{k})$ : During the reception of the WUB in case of $\operatorname{MEES}(n, k)$, when the ULP-MCU detects the first ' 1 ' it checks if its position is correct to send the interrupt, otherwise it does not send the interrupt. In both cases the ULP-MCU will be early shutdown. From (26), the average latency denoted $\bar{L}_{M E E S}$ is expressed as:

$$
\bar{L}_{M E E S}=T_{b}\left(l_{\text {preamble }}+\bar{l}_{M E E S}\right) .
$$

2) Average latency of the WUB and data exchange: The average latency of successfully receiving the WUB followed by the data exchange is denoted $\bar{L}_{\text {total }}$, and it can be expressed as:

$$
\bar{L}_{\text {total }}=n_{t x} \bar{L}_{x}+T_{d a t a}+T_{a c k},
$$

with $\bar{L}_{x}$ can be, according to the used coding scheme, either $\bar{L}_{\text {uncoded }}, \bar{L}_{M E}$ or $\bar{L}_{M E E S}$, that were defined in (32), (33) or (34). When considering MEES, the average latency of the WUB and data exchange model is valid if the transmitter sends the data just after sending the ' 1 ' 'in the WUB.

\section{EXPERIMENTAL EVALUATION}

\section{A. Experimental testbed}

Both $\operatorname{ME}(7,3)$ and $\operatorname{MEES}(7,3)$ have been implemented on the WuR designed in [21] and described in Section II. Different metrics have been evaluated: missed detection rate, WDR, average power consumption and average latency. This WuR is based on a non-coherent detector and its sensitivity was measured to be around $-45 \mathrm{dBm}$.

The transmitter sends to the WuR a WUB that contains a preamble and an address, the ULP-MCU of the WuR performs an address matching to check if it is receiving the correct address and then sends an interrupt to wake up the main receiver. The preamble contains three ones bits. The length of the sent address depends on the use or not of the coding.

Fig. 4 shows the format of the WUB by an oscilloscope when the address 0 b101 is sent in the three cases (uncoded (0b101), ME(7,3) (0b0010000) and MEES(7,3) (0b0010000)) and the respective latency before transmitting an interrupt to the main node. The address is preceded by a 3-bit preamble and one bit at low level that allows to trigger the ULP-MCU and start decoding from zero crossing. 


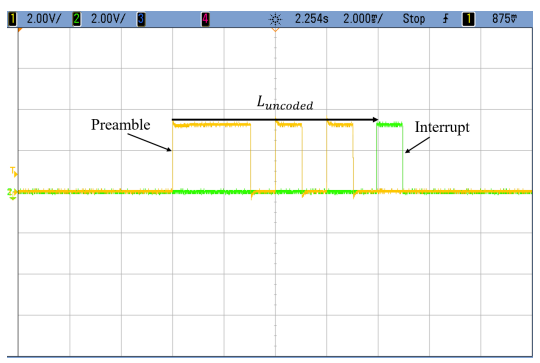

(a) Uncoded

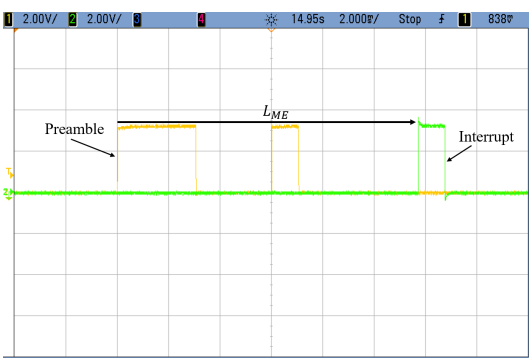

(b) $\mathrm{ME}$

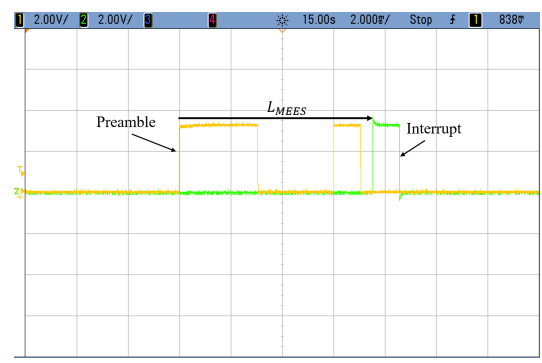

(c) MEES

Fig. 4. The WUB format when sending the address 0 b101 with different schemes.

It can be seen on Fig. 4(c) that for the MEES scheme, the interrupt is sent immediately after the first ' 1 ' is received, contrary to uncoded and ME schemes, illustrated by Fig. 4(a) and Fig. 4(b), respectively.

In Section V-B, the missed detection rate is evaluated as a function of all possible addresses that could be sent w.r.t two different transmission power levels. Then, the WDR is evaluated in Section $\mathrm{V}-\mathrm{C}$ in an anechoic chamber with different transmission power levels ranging from $-13 \mathrm{dBm}$ to $-19.5 \mathrm{dBm}$. Results of the average power consumption and the average latency are also respectively presented in Section V-D and Section V-E.

\section{B. Missed detection rate evaluation}

The transmitter and the receiver were connected by RF cable as shown in Fig. 5, thus the transmitted power is the same as the received power when considering a negligible loss of the cable. The missed detection rate represents the total number of bits decoded wrongly by the ULP-MCU over the total number of bits sent in the WUB address. The WUB is transmitted each $250 \mathrm{~ms}$ and in average $10^{5}$ times for each possible address.

Fig. 6 shows the experimental evaluation of the missed detection rate as a function of the address sent with and without MEES. Fig. 6(a) and Fig. 6(b) show the missed detection rate when the received power is close to the sensitivity and equals to $-45 \mathrm{dBm}$ and $-44 \mathrm{dBm}$, respectively. It can be seen that in

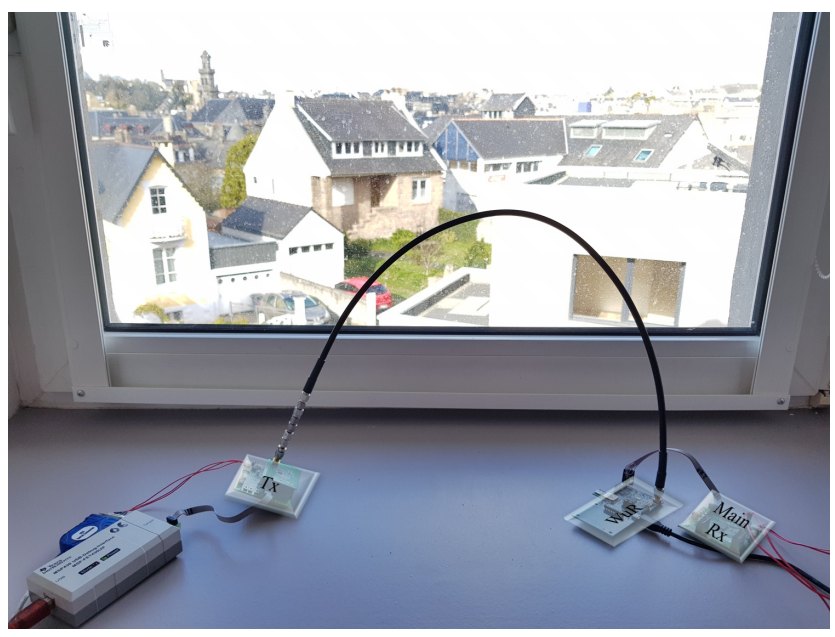

Fig. 5. Experimental testbed for missed detection rate evaluation.

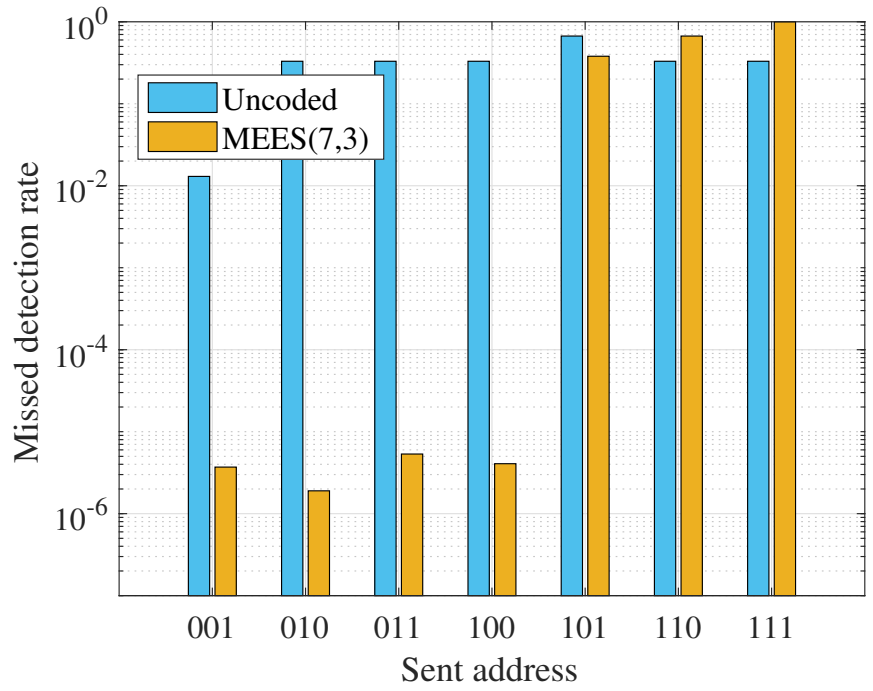

(a) Received power $-45 \mathrm{dBm}$

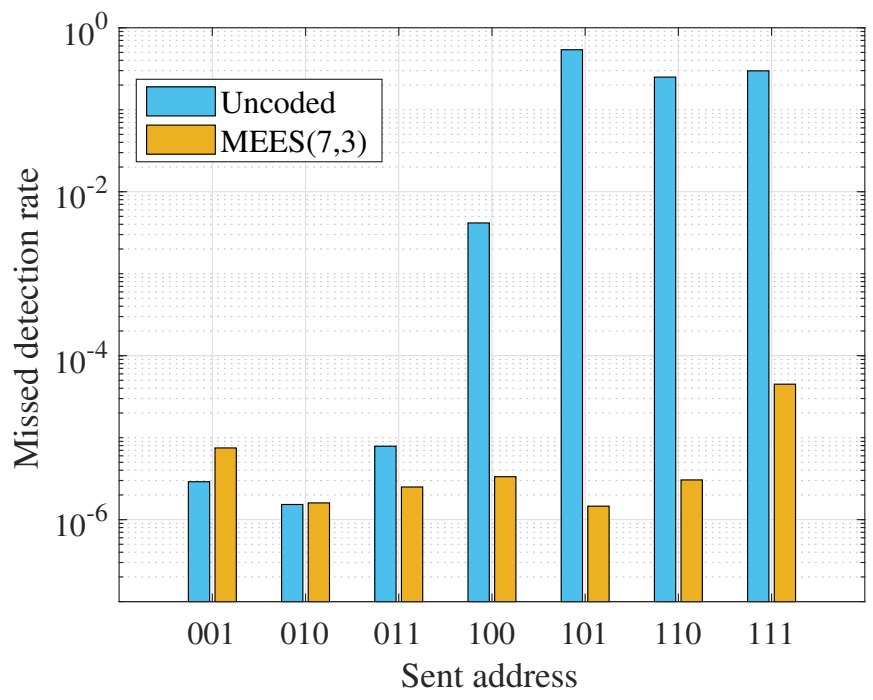

(b) Received power $-44 \mathrm{dBm}$

Fig. 6. Missed detection rate as a function of the sent address.

both cases the missed detection rate when applying $\operatorname{MEES}(7,3)$ is better than when no code is used. It can also be seen that when the first ' 1 ' is close to the preamble there are more errors; that is probably due to the inter-symbol interference between the preamble and the address. Thus the performance depends on the address sent for both schemes. To reduce the 
bit error rate it is preferable to use the addresses in which the ' 1 ' is far from the preamble.

Without coding the average missed detection rate is equal to 0.33 and 0.16 when the transmission power is equal to $-45 \mathrm{dBm}$ and $-44 \mathrm{dBm}$, respectively. However, when applying MEES the average missed detection rate is equal to 0.29 and $10^{-5}$ when the transmission power is equal to $-45 \mathrm{dBm}$ and $-44 \mathrm{dBm}$, respectively.

The missed detection rate could be further improved through a premble filtering mechanism [31].

\section{WDR evaluation}

Experimental evaluation of the $W D R$ was realized in the anechoic chamber as shown in Fig. 7(a). The transmitter and the WuR were placed at a distance of $1 \mathrm{~m}$.

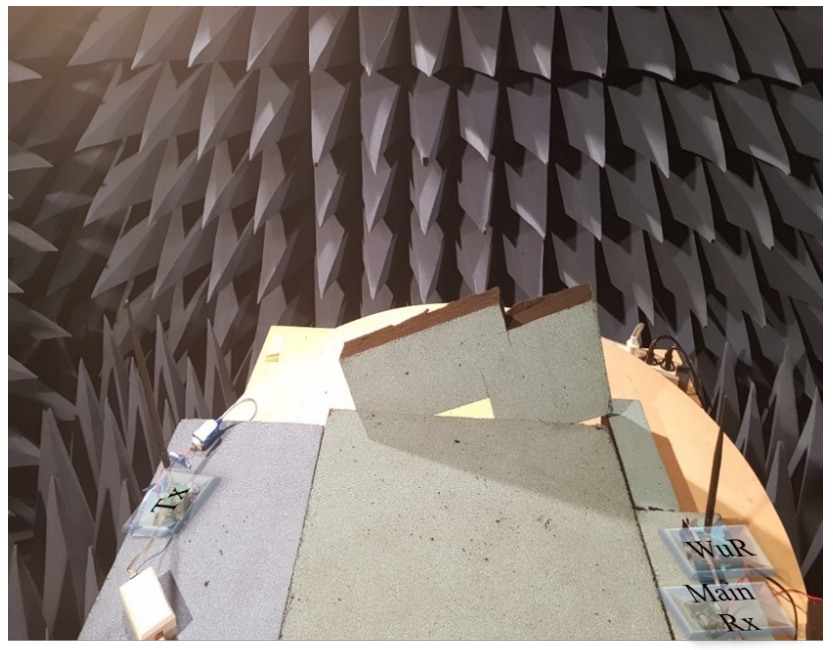

(a) Experimental testbed in anechoic chamber.

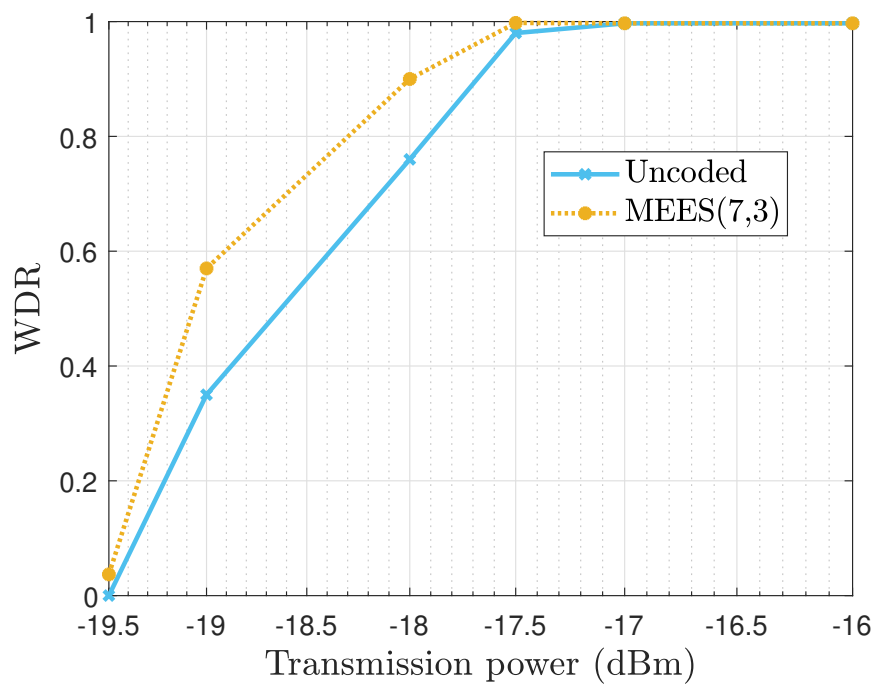

(b) Experimental $W D R$ as a function of the transmission power.

Fig. 7. Experimental testbed and results of PDR.

Fig. 7(b) shows the $W D R$ in the anechoic chamber as a function of different transmission power levels ranging from $-19.5 \mathrm{dBm}$ to $-16 \mathrm{dBm}(-19.5 \mathrm{dBm}$ is close to the sensitivity of $-45 \mathrm{dBm}$ at a range of $1 \mathrm{~m})$. The $W D R$ is measured for 7215 WUBs transmitted at a rate of 4 packets/second. For each transmission power, the $W D R$ is measured for all possible addresses that could be sent in the WUB with $\operatorname{MEES}(7,3)$ and without coding and then the average $W D R$ is considered. It can be seen that beyond a transmission power of $-17 \mathrm{dBm}$ the $W D R$ is the same with and without coding and it is equal to $100 \%$. At a transmission power lower than $-17 \mathrm{dBm}$, the $W D R$ when using $\operatorname{MEES}(7,3)$ is better than without coding. For example, at a transmission power of $-19 \mathrm{dBm}$ a gain of $22 \%$ of WUBs successfully received can be achieved when applying $\operatorname{MEES}(7,3)$.

\section{Average power consumption evaluation}

The average power consumption is evaluated by considering the models presented in Section IV-B, and a network of 10 nodes is considered. For the power consumed by the transmitter, the S2-LP ultra low power, high performance, sub$1 \mathrm{GHz}$ transmitter from ST [32] is considered. According to the data-sheet the power consumption $P_{t x_{1}}$ is equal to $13.8 \mathrm{~mW}$ when transmitting a ' 1 ' at $10 \mathrm{dBm}$ and when transmitting a ' 0 ' the power amplifier is turned off, thus consuming a $P_{t x_{0}}$ equal to $420 \mu \mathrm{W}$, with a delay of $70 \mu$ s for switching from ' 0 ' to ' 1 ', which is acceptable for a bitrate of $1 \mathrm{kbps}$. According to the same data-sheet, $P_{t x}=13.8 \mathrm{~mW}$ (as the main transceiver is always turned on during all data transmission), $P_{r x}=8.64 \mathrm{~mW}$ and $P_{s}=0.8 \mu \mathrm{W}$. For the $\mathrm{WuR}$, the $\mathrm{WuR}$ that was designed in [21] is considered. It works at a rate of $1 \mathrm{kbps}$ with a measured power consumption of $1.83 \mu \mathrm{W}$ in always listening mode and $284 \mu \mathrm{W}$ when receiving and processing the WUB with the ULP-MCU (PIC12LF1552 from Microchip [33]). The duration of data and acknowledgement are $T_{\text {data }}=4.09 \mathrm{~ms}$ (considering 128 bytes at $250 \mathrm{kbps}$ [30]) and $T_{a c k}=0.04 \mathrm{~ms}$, respectively.

Fig. 8(a) shows the average power consumption of successfully transmitting the WUB when applying the ME coding with $\mathrm{k}=3$ and $\mathrm{k}=4$, and without coding, as a function of $\lambda$ (we do not talk about MEES at the transmission as the early shutdown concerns only the WuR). The number of times the WUB is transmitted to be successfully received depends on the $W D R$. The measured $W D R$ in the previous section is considered at a power transmission of $-19 \mathrm{dBm}$ at a range of $1 \mathrm{~m}$, that is equivalent to a theoretical power transmission of $10 \mathrm{dBm}$ and a range of $28 \mathrm{~m}$. It can be seen from Fig. 8(a) that the power consumed to send the WUB without coding when $\mathrm{k}=3$ and $\mathrm{k}=4$ can be respectively up to green 1.79 and 1.85 times more, compared to when using coding. Even if the WUB length is increased with ME coding, more '0' are transmitted than '1', and transmitting ' 0 ' does not consume a lot with the chosen transmitter. Furthermore, as ME coding has the best $W D R$, and thus needs to re-transmit less WUBs than uncoded scheme to be successfully received by the WuR.

Fig. 8(b) shows the average power consumption of the WuR without coding and when applying ME and MEES coding with $\mathrm{k}=3$ and $\mathrm{k}=4$ as a function of $\lambda$. It can be seen that when using the ME coding, the WuR consumes more compared to without coding, this is due to the fact that the ULP-MCU stays ON for 


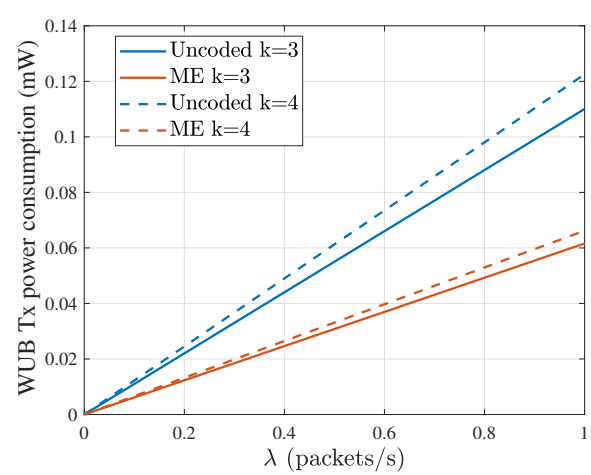

(a) WUB transmission

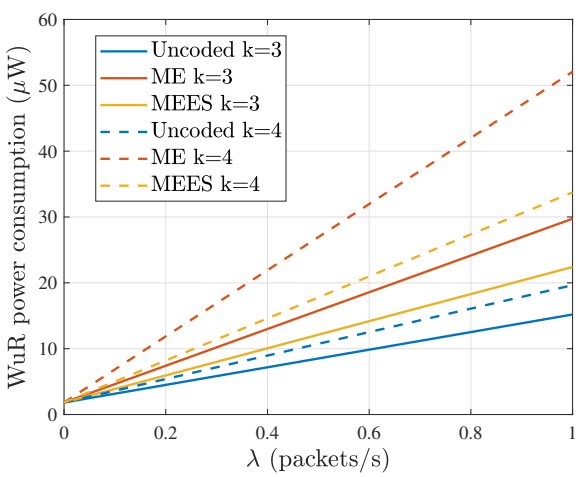

(b) WuR

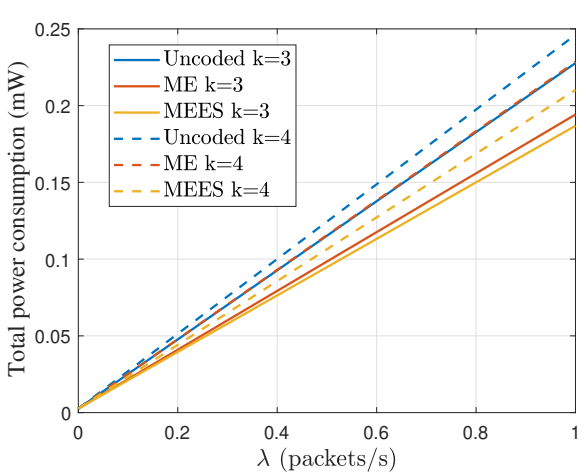

(c) Total

Fig. 8. Average power consumptions of transmitting the WUB, WuR and total as a function of $\lambda$.

longer time to process the received address. However, when using MEES the WuR consumes less than ME as the ULPMCU is shutdown right after receiving the first ' 1 . At a rate of 1 packet/s and when applying MEES, the WuR consumes up to 1.33 and 1.54 times less over ME coding with $\mathrm{k}=3$ and $\mathrm{k}=4$, respectively. Meanwhile, it still consumes 1.47 and 1.72 times more with $\mathrm{k}=3$ and $\mathrm{k}=4$, respectively compared to uncoded WuR.

Fig. 8(c) shows the average total power consumption of the node. The power consumption is evaluated as a function of $\lambda$ without coding and when applying ME and MEES coding with $\mathrm{k}=3$ and $\mathrm{k}=4$. It can be seen that the average total power consumption of the node with uncoded scheme is higher than when using coding, as the energy consumption of the WuR is far less than the transmission of the WUB. It appears that with uncoded scheme the node consumes up to 1.22 and 1.17 times more (corresponding to an increase of almost $18 \%$ and $14 \%$ ) with $\mathrm{k}=3$ and $\mathrm{k}=4$, respectively compared to MEES scheme. Furthermore, MEES consumes 1.03 and 1.09 times less (corresponding to a reduction of almost 3\% and 8.2\%) with $\mathrm{k}=3$ and $\mathrm{k}=4$, respectively over ME scheme.

\section{E. Latency evaluation}

1) WUB exchange latency: The theoretical latency obtained by (32), (33) and (34) in Section IV-C are compared with experimental measurements in this section.

Fig. 9 shows the average latency of the WUB exchange when considering all possible symbols of $\mathrm{k}=3$ bits and $\mathrm{k}=4$ bits. Both experimental and theoretical results are shown. It can be seen that there is a small difference between the theoretical and experimental results, this difference is due to the fact that the theoretical model does not take into consideration the processing time done by the ULP-MCU before sending the interrupt to the main radio. It can be seen that when applying ME coding the latency is more important as the ULP-MCU should process the whole WUB. When applying MEES, the experimental average latency is increased by $1.02 \mathrm{~ms}$ when $\mathrm{k}=3$ bits compared to the uncoded symbol. However, it is reduced by $2.98 \mathrm{~ms}$ than ME coding. When the size of the symbol is 4 bits of MEES, the experimental average latency is reduced by $6.91 \mathrm{~ms}$ over the ME coding, but it is increased by $4.09 \mathrm{~ms}$ over the uncoded scheme.

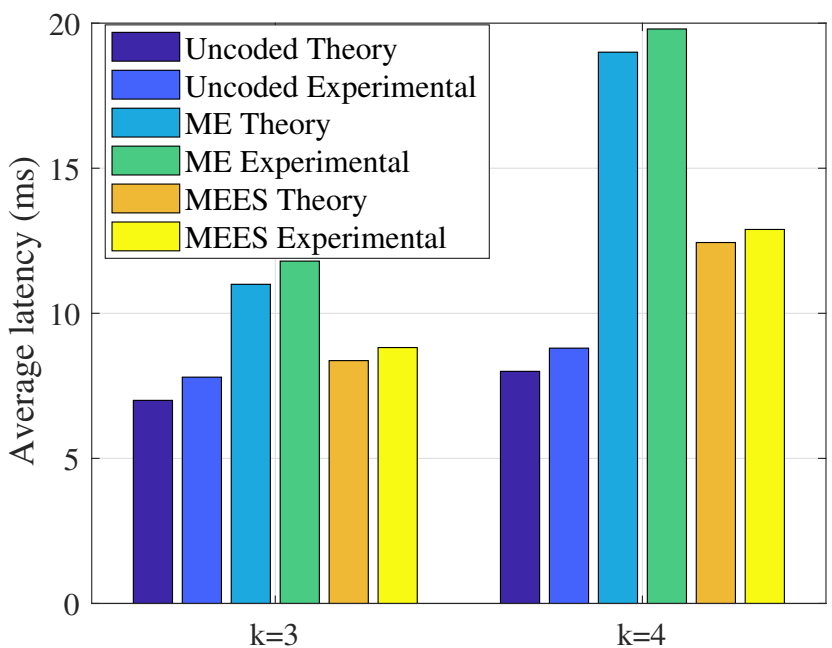

Fig. 9. Average latency of WUB exchange over k.

2) Total latency: The average total latency of the WUB and data exchange derived in (35) is evaluated in Fig. 10 for $\mathrm{k}=3$ and $\mathrm{k}=4$. It can be seen that when applying MEES the average total latency is reduced compared to both uncoded and ME coding schemes. As the WDR is improved when applying coding, there are less re-transmitted packets compared to uncoded scheme. Moreover, the average latency is reduced with MEES because the ULP-MCU is early shutdown, and because the duration of data is far less than the duration of the WUB due to the difference in bitrate. Therefore, the average total latency is reduced with MEES. It appears that for $\mathrm{k}=3$, the average total latency is reduced by $3.06 \mathrm{~ms}$ with MEES compared to uncoded scheme, and it is reduced by $2.89 \mathrm{~ms}$ compared to ME scheme. For $\mathrm{k}=4$, the total latency is reduced by $0.37 \mathrm{~ms}$ with MEES compared to uncoded scheme, and it is reduced by $7.21 \mathrm{~ms}$ over ME scheme.

\section{CONCLUSION}

In this paper a channel coding scheme suitable to the Wakeup Receivers (WuRs) is proposed in the purpose to improve the WuR sensitivity while maintaining a low energy consumption. Leveraging minimum energy coding, our proposed coding scheme offers the possibility to early shutdown the ULP-MCU 


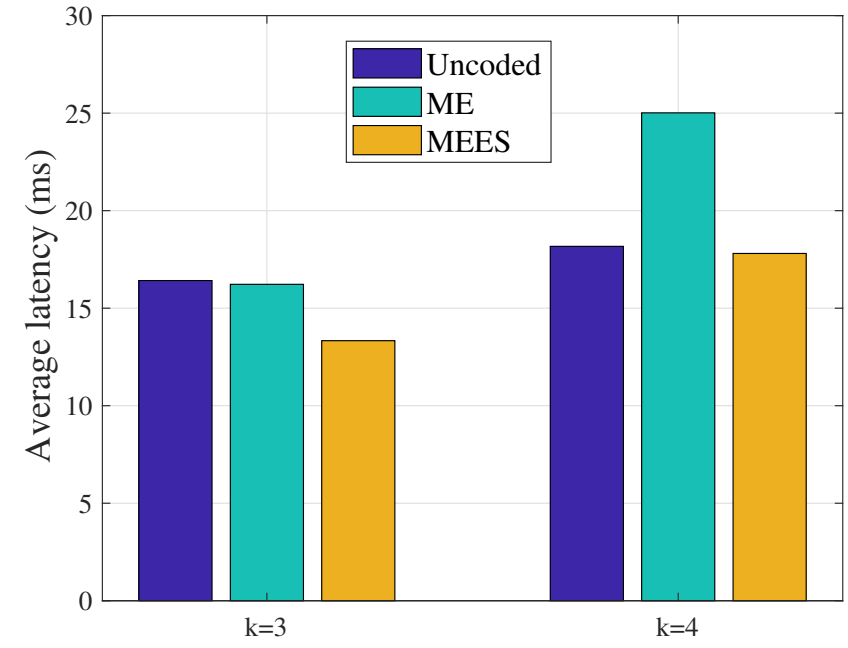

Fig. 10. Average total latency over k.

of the WuR, and is named Minimum Energy Coding Early Shutdown (MEES). The bit-error probability of MEES was derived and monte-carlo simulations were presented. Results show that MEES can either increase the range under the same energy consumption or reduce the energy consumption under the same BER. Average power consumption models of a sensor node were derived within a network. Then, the proposed MEES was implemented in a WuR testbed and the experimental evaluation of the reliability was done in anechoic chamber. Results show the benefits of MEES in improving the successful received packets up to $22 \%$. The evaluation of average power consumption demonstrates a reduction about $18 \%$ with MEES compared to uncoded scheme. The evaluation of the latency was also performed proving the efficiency of MEES. Future works will investigate the combination of MEES scheme with other coding schemes.

\section{ACKNOWLEDGMENT}

This work is part of the project Wake-Up funded by the French National Research Agency (ANR).

\section{REFERENCES}

[1] I. F. Akyildiz, W. Su, Y. Sankarasubramaniam, and E. Cayirci, "A survey on sensor networks," IEEE Communications Magazine, vol. 40, no. 8, pp. 102-105, 2002.

[2] J. Suh and M. Horton, "Powering sensor networks," IEEE Potentials, vol. 23, no. 3, pp. 35-38, 2004.

[3] A. Bachir, M. Dohler, T. Watteyne, and K. K. Leung, "MAC Essentials for Wireless Sensor Networks," IEEE Communications Surveys Tutorials, vol. 12, no. 2, pp. 222-248, 2010.

[4] R. C. Carrano, D. Passos, L. C. Magalhaes, and C. V. Albuquerque, "Survey and taxonomy of duty cycling mechanisms in wireless sensor networks," IEEE Communications Surveys and Tutorials, vol. 16, no. 1, pp. 181-194, 2014.

[5] M. M. Islam, Z. Al Nazi, A. B. M. A. Hossain, and M. M. Rana, "Data Prediction in Distributed Sensor Networks Using Adam Bashforth Moulton Method," Journal of Sensor Technology, vol. 08, no. 02, pp. 48-57, 2018.

[6] F. Ait Aoudia, M. Magno, M. Gautier, O. Berder, and L. Benini, "Analytical and Experimental Evaluation of Wake-Up Receivers Based Protocols," in IEEE Global Communications Conference (GLOBECOM), 2016, pp. $1-7$.
[7] J. Oller, I. Demirkol, J. Casademont, J. Paradells, G. U. Gamm, and L. Reindl, "Has Time Come to Switch from Duty-Cycled MAC Protocols to Wake-Up Radio for Wireless Sensor Networks?" IEEE/ACM Transactions on Networking, vol. 24, no. 2, pp. 674-687, 2016.

[8] M. Magno, S. Marinkovic, B. Srbinovski, and E. Popovici, "Wake-up radio receiver based power minimization techniques for wireless sensor networks: A review," Microelectronics Journal, vol. 45, no. 12, pp. 1627-1633, 2014.

[9] N. E. H. Djidi, A. Courtay, M. Gautier, and O. Berder, "Adaptive relaying for wireless sensor networks leveraging wake-up receiver," in IEEE International Conference on Electronics, Circuits and Systems (ICECS), 2018, pp. 797-800.

[10] F. Ait Aoudia, M. Gautier, M. Magno, M. Le Gentil, O. Berder, and L. Benini, "Long-short range communication network leveraging LoRa' ${ }^{\mathrm{TM}}$ and wake-up receiver," Microprocessors and Microsystems, vol. 56, pp. 184-192, 2018.

[11] R. Piyare, A. L. Murphy, C. Kiraly, P. Tosato, and D. Brunelli, "Ultra Low Power Wake-Up Radios: A Hardware and Networking Survey," IEEE Communications Surveys \& Tutorials, vol. 19, no. 4, pp. 2117 2157, 2017.

[12] D. Ghose, A. Frøytlog, and F. Y. Li, "Enabling early sleeping and early data transmission in wake-up radio-enabled IoT networks," Computer Networks, vol. 153, pp. 132-144, 2019.

[13] V. Rakovic, R. Adamovski, A. Risteski, and L. Gavrilovska, "Improving Energy Efficiency and Reliability in WuR-Based IoT Systems: An Error Correction Approach," in Wireless Personal Communications, 2020.

[14] Q. Tang, S. Gupta, and L. Schwiebert, "BER performance analysis of an on-off keying based minimum energy coding for energy constrained wireless sensor applications," in IEEE International Conference on Communications (ICC), 2005, pp. 2734-2738.

[15] F. Z. Djiroun and D. Djenouri, "MAC Protocols With Wake-Up Radio for Wireless Sensor Networks: A Review," IEEE Communications Surveys \& Tutorials, vol. 19, no. 1, pp. 587-618, 2017.

[16] J. M. Rabaey, J. Ammer, T. Karalar, S. Li, B. Otis, M. Sheets, and T. Tuan, "PicoRadios for wireless sensor networks: The next challenge in ultra-low power design," in IEEE International Solid-State Circuits Conference, 2002, pp. 2001-2002.

[17] C. Bryant and H. Sjoland, "A $2.45 \mathrm{GHz}, 50 \mu \mathrm{W}$ wake-up receiver frontend with $-88 \mathrm{dBm}$ sensitivity and $250 \mathrm{kbps}$ data rate," in European Solid State Circuits Conference (ESSCIRC), 2014, pp. 235-238.

[18] P. N. Thanh, K. N. Tuan, and X. M. Dong, "A 100- $\mu \mathrm{W}$ wake-up receiver for UHF transceiver," in International Conference on IC Design and Technology (ICICDT), 2016, pp. 1-4.

[19] S. Moazzeni, M. Sawan, and G. E. R. Cowan, "An Ultra-Low-Power Energy-Efficient Dual-Mode Wake-Up Receiver," vol. 62, no. 2, pp. 517526, 2015.

[20] S. Bdiri and F. Derbel, "An Ultra-Low Power Wake-Up Receiver for Real-time constrained Wireless Sensor Networks," in SENSOR, 2015 , pp. 612-617.

[21] M. Magno, V. Jelicic, B. Srbinovski, V. Bilas, E. Popovici, and L. Benini, "Design, Implementation, and Performance Evaluation of a Flexible Low-Latency Nanowatt Wake-Up Radio Receiver," IEEE Transactions on Industrial Informatics, vol. 12, no. 2, pp. 633-644, 2016.

[22] P.-H. P. Wang, H. Jiang, L. Gao, P. Sen, Y.-H. Kim, G. M. Rebeiz, P. P. Mercier, and D. A. Hall, "A $6.1 \mathrm{nW}$ Wake-Up Receiver Achieving -80.5 dBm Sensitivity Via a Passive Pseudo-Balun Envelope Detector," IEEE Solid-State Circuits Letters, vol. 1, no. 5, pp. 134-137, 2018.

[23] J. Moody, P. Bassirian, A. Roy, N. Liu, N. S. Barker, B. H. Calhoun, and S. M. Bowers, "Interference Robust Detector-First Near-Zero Power Wake-Up Receiver," IEEE Journal of Solid-State Circuits, vol. 54, no. 8, pp. 2149-2162, 2019.

[24] H. Jiang, P. H. P. Wang, L. Gao, C. Pochet, G. M. Rebeiz, D. A. Hall, and P. P. Mercier, "A 22.3-nW, $4.55 \mathrm{~cm}^{2}$ Temperature-Robust Wake-Up Receiver Achieving a Sensitivity of $-69.5 \mathrm{dBm}$ at $9 \mathrm{GHz}$," IEEE Journal of Solid-State Circuits, vol. 55, no. 6, pp. 1530-1541, 2020.

[25] L. W. Couch, Digital and Analog Communication Systems 6th ed. Prentice Hall, 2001

[26] Q. Chen, L. Wang, P. Chen, and G. Chen, "Optimization of Component Elements in Integrated Coding Systems for Green Communications: A Survey," IEEE Communications Surveys \& Tutorials, vol. 21, no. 3, pp. 2977-2999, 2019.

[27] P. Zhang, F. M. J. Willems, and L. Huang, "Investigations of noncoherent OOK based schemes with soft and hard decisions for WSNs," in Conference on Communication, Control, and Computing (Allerton), 2011, pp. 1702-1709. 
[28] N. S. Mazloum and O. Edfors, "Performance analysis and energy optimization of wake-up receiver schemes for wireless low-power applications," IEEE Transactions on Wireless Communications, 2014.

[29] M. Schwartz, W. R. Bennett, and S. Stein, Communication systems and techniques. John Wiley \& Sons, 1995.

[30] M. Zhang, D. Ghose, and F. Y. Li, "Does Wake-Up Radio Always Consume Lower Energy Than Duty-Cycled Protocols?" in Vehicular Technology Conference (VTC-Fall), 2017, pp. 1-5.

[31] N. E. H. Djidi, M. Gautier, A. Courtay, and O. Berder, "Enhancing wakeup receivers reliability through preamble filtering and minimum energy coding," in IEEE International Symposium on Circuits and Systems (ISCAS), 2021, pp. 1-5.

[32] ST, Ultra-low power high performance sub-1 GHz transceiver Datasheet, 2019, accessed: 2020-05-19. [Online]. Available: https://www.st.com/content/st_com/en.html

[33] Microchip, "PIC12LF1552 Datasheet," 2015, accessed: 2020-07-08. [Online]. Available: http://www.microchip.com

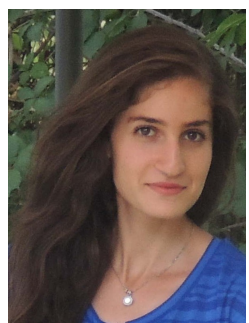

Nour El Hoda DJIDI received the engineering degree in electronics from Ecole Nationale Polytechnique d'Alger, and a masters degree of embedded systems and information processing from university Paris Saclay in 2018. She is currently a Ph.D student at the University of Rennes 1, IRISA laboratory. She was a visitor Ph.D student at ETHZ between October and December 2019.

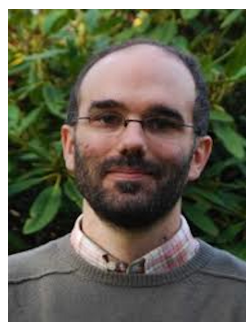

Matthieu Gautier received the Engineering and M.Sc. degrees in electronics and signal processing engineering from the ENSEA Graduate School, the Ph.D. degree from Grenoble-INP in 2006, and the French HDR in 2019. From 2006 to 2011, he was a Research Engineer with Orange labs, INRIA and CEA-LETI Laboratory. He is currently an Associate Professor with the University of Rennes and the IRISA Laboratory since 2011. His research activities are in the two complementary fields of embedded systems and digital communications for energy efficient wireless bioadjust systems. He has co-authored over 60 peer reviewed papers in international journals or conferences and has participated in numerous collaborative projects funded by either the European Union or French National Research Agency.

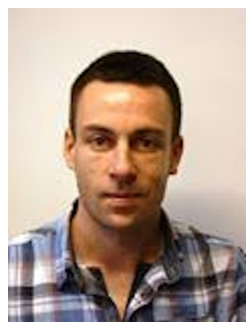

Antoine Courtay received the Ph.D. degree in Electronics \& Computer Sciences from the University of Bretagne Sud, Lorient, France, in 2008. From 2008 to 2009 he was an assistant professor at the University of Bretagne Sud, Lorient and at the LabSTICC Laboratory. From 2009 to 2010 he was a post-doctoral researcher at the University of Nice Sophia Antipolis, Nice and at the LEAT Laboratory. From 2010 to 2012 he was an electronic engineer at the LBMS Laboratory and ENIB. Since September 2012, he joined the University of Rennes 1 - ENSSAT and IRISA Laboratory as an associate professor. His research activities currently concern the implementation of energy efficient algorithms targeting wireless sensor networks platforms.

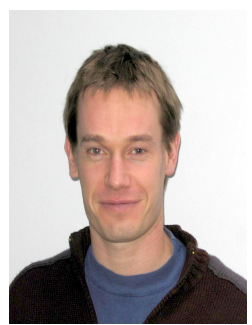

Olivier Berder received the Ph.D. degrees in electrical engineering from the University of Bretagne Occidentale, Brest, in 2002. After a post-doctoral position at Orange Labs, in March 2005 he joined University of Rennes 1 where he is currently a Full Professor at IUT Lannion, and the leader of GRANIT team of IRISA Laboratory. His research interests focus on multiantenna systems and cooperative techniques for energy efficient mobile communications and wireless sensor networks. He has co-authored more than 80 peer reviewed papers in international journals or conferences and has participated in numerous collaborative projects founded by either European Union or French National Research Agency, mainly related to Wireless Sensor Networks. 